\title{
CURRENT STATUS OF THE ASTROMETRIC CAPABILITIES OF THE HUBBLE SPACE TELESCOPE FINE GUIDANCE SENSORS
}

\author{
W. H. Jefferys*, G. F. Benedict*, R. L. Duncombe**, O. G. Franz***, L. W. \\ Fredrick****, T. Gerard*****, P. D. Hemenway*, B. McArthur*, J. \\ McCartney*, E. Nelan*, P. J. Shelus*, D. Story*, W. van Altena*****, L. \\ Wasserman***, A. Whipple*, J. Whitney**** \\ *Department of Astronomy, **Center for Space Research, University of Texas, \\ Austin, TX; ***Lowell Observatory, Flagstaff, AZ; ****Dept. of Astronomy, \\ University of Virginia, Charlottesville, VA; *****Dept. of Astronomy, Yale \\ University, New Haven, CT.
}

\begin{abstract}
The Fine Guidance Sensors (FGSs) are the instrument of choice for most astrometric measurements with the Hubble Space Telescope (HST). The observed amount of spherical aberration in the Ritchey Chretien optical system does not affect positional measurements with perfectly aligned FGSs because they are interferometers. The FGSs combine wavefronts from points in the exit pupil with other points which are at the same radial distance from the optical axis. Asymmetric aberrations such as coma and astigmatism do affect the measured positions. The current knowledge of the HST wavefront error, the FGS operation and the implications for milliarcsecond relative astrometry are discussed. It is still planned to use the HST to tie the HIPPARCOS and VLBI Reference Frames together at the few milliarcsecond level.
\end{abstract}

\section{Introduction}

On the morning of 24 April 1990, STS-31 carried the Hubble Space Telescope into space. It was placed into orbit the following day with an inclination of 28.5 degrees, perigee of 331 nautical miles and apogee of 332 nautical miles; all very close to nominal.

Of the six scientific instruments which comprise the instrumental package of the Hubble Space Telescope (HST), only the Fine Guidance Sensors (FGSs) will be discussed. Three Fine Guidance Sensors are associated with the HST. These interferometric devices, as part of the Pointing Control System, fix the orientation of HST with respect to known stars, or may be used for astrometric measurements. The location of the three FGS fields of view in the focal plane of HST are shown in figure 1. The design aspects of the Fine Guidance Sensors as well as their operational and observational modes are completely described in references 7 and 8 given in the bibliography.

Figure 2 shows the fields of view of the three FGSs with respect to the optical axis.

The FGSs use Koester's prisms to combine two images of the exit pupil which are reversed with respect to each other. Two photomultiplier tubes measure the light on either side of the interference pattern; call these outputs A and B. A Fine Error Signal (FES) is produced by measuring $(A-B) /(A+B)$. A hypothetical plot of the FES as a function of the position offset from the interferometer "null" position is given in figure 2 . The null position is the center of the pattern where the transfer function passes through zero. 
This paper presents the results of consistant sets of transfer functions observed under a variety of conditions. Affects of the observed vehicle jitter, the telescope optics problems, and possible internal FGS problems are discussed.

\section{The Observations}

We have obtained transfer scans in all FGSs, but with limited data. We have not obtained a consistent set of data (different magnitude stars, jittered and non-jittered conditions, same secondary mirror position, etc.) in POS mode with full aperture in any FGS and in POS mode with $2 / 3$ aperture in any FGS except FGS3. However, fine lock has been achieved for guiding in all three FGSs on bright stars (above 13th magnitude).

The HST ground system is slow to respond to new input. From the time an observation is first formulated, written, submitted, approved and scheduled by the Space Telescope Science Institute, at least 4 to 6 weeks will elapse before the observation is integrated into the timeline and actually performed.

\subsection{THE DATA}

A series of Transfer scans were obtained on sets of stars during two programs. The first program was a series of secondary mirror position changes to characterize the change of image structure in the Planetary Camera and the Faint Object Camera frames as a function of focal position. At each mirror position, WFPC and FOC images were taken (except when guide stars were lost due to vehicle jitter) and transfer scans were performed with the FGSs on a 9th , a 12th, and a 15th magnitude star. Each star was scanned five times, providing reproducibility checks. Scans were performed with both clear filter and 2/3 apertures. These scans provided the first consistent set of data on the behavior of the three FGSs. All data were taken while guiding in Coarse Track.

The second series of positions was obtained in an attempt to determine the optical field angle distortions (OFAD) within each pickle. The observations were made in NGC188 where the relative positions are known from ground based observations at the 0.01 arcsecond level of accuracy (rms). Ultimately, the OFAD will be determined by a priori measurements and a full plate overlap solution at the 0.002 arcsecond level, but the initial characterization of the OFAD is done with respect to the ground-based measurements to get first-order values for the OFAD coefficients.

\subsection{REVIEW OF THE DATA--SYMPTOMS, PROBLEMS, AND POSSIBLE CAUSES}

Three areas that contribute to the FGS problems may be identified: Vehicle jitter, OTA (primary and/or secondary mirror) misfigurement and misalignment, and possible internal FGS problems.

2.2.1 Vehicle Jitter. When the Telescope is guided in Coarse Track, the sum of the PMT counts from the four quadrants of the 5 arcsecond square field stop (IFOV) are used to determine the centroid position of the guide stars, as the stop is "nutated" around the star. The position is updated 4 times per second while using all of the data from the previous full second; therefore, the Coarse Track information can be used to estimate how much the 
vehicle line of sight is moving as a function of time. The thermal variation of the solar arrays as the telescope moves from orbit day into orbit night causes the pointing of the telescope to "jitter". The peak-to-peak excursions after the day/night transition are on the order of .2 arcseconds while the "quiet time" peak-to-peak excursions are on the order of .050 arcseconds. A dominant frequency of $0.1 \mathrm{~Hz}$ can be clearly seen with $0.6 \mathrm{~Hz}$ also present. A digital filtering algorithm is being put into the Pointing Control System Flight Software to attempt to remove the $0.1 \mathrm{~Hz}$ jitter. If the $0.6 \mathrm{~Hz}$ jitter is driven by the $0.1 \mathrm{~Hz}$, then the $0.6 \mathrm{~Hz}$ jitter will be reduced also. We hope the jitter will be significantly reduced, since we believe it to be perturbing the transfer scan data (and hence varying position measurements) with the additional possibility of contributing to the loss of Fine Lock on guide stars fainter than 13th magnitude.

Consecutive scans of the same star with the same FGS show large variations in the character of the Transfer Functions. Dr. Gerald Nurre has simulated the effect of jitter (input stimulus) at $0.1 \mathrm{~Hz}$ and $0.6 \mathrm{~Hz}$ on an ideal transfer function, while varying the phase of the input stimulus with respect to the phase of the transfer scan. The variation of the signature in some of his simulations show induced characteristics which are strikingly similar to the observations from one scan to another.

\subsubsection{OTA Misalignments/Possible Internal FGS Problems}

i. Figure 3 shows transfer scans for FGS2, 1, and 3 in $x$ and $y$ on the same star with the same aperture (2/3). The ordinate is the normalized Fine Error Signal. The separation between the tick marks is either 0.1 or 0.2 unitless numbers. The expected theoretical amplitude for a perfect FGS is between 0.7 and 0.8. The amplitudes of FGS3 are close to nominal, whereas the amplitudes of the other two are significantly reduced. The abscissa is in arcseconds where the total data plotted is about one or about two arcseconds depending on the individual plots. The width of the ideal transfer function from the positive peak to the negative peak is about 40 milliarcseconds and can be used to estimate the scales of the individual graphs. The plots have been auto-scaled and are reproduced from the online data extraction system. The different FGS scans were observed at different times. The transfer scans taken so far are predominantly taken in the center of the pickle. Clear differences between the transfer scans indicate that FGS to FGS differences exist. The $x$ coordinate in FGS2 corresponds to the y coordinates in FGSs 1 and 3, while the y coordinate in FGS2 corresponds to the $x$ coordinates in FGSs 1 and 3 . If a star is double, then the component separations which show up in $x$ and/or $y$ in FGS2 will show up in the reversed coordinates in the other two FGSs. Therefore, if a scan which appears as a "W" were due to a double star in the y component of FGS2, then it would show up as a "W" in $x$ in FGS1 and FGS3. While structure exists in the odd FGSs, it is unlikely to be due to a double star because of the different nature of the transfer functions. 12th magnitude stars were used for this comparison because comprehensive consistant data on brighter stars do not yet exist.

ii. Focus Shift. A comparison has been made between transfer function scans of the same star at two slightly different focus settings of the secondary mirror. The position difference between the two settings was 10 microns along the optical axis. Within the noise, no difference between the two scans can be determined. However, the focus position difference is so slight that nothing can be said about the change in the characteristic of the transfer function for widely separated focus settings. Still, the result indicates that the system is stable after a mirror move.

iii. Magnitude Comparison. A comparison has been made between scans with the same FGS on a 12th magnitude and a 15th magnitude star. Except for the noise, the characteristics appear to be the same. However, the difficulty of "picking out" the transfer function from the noise in the fainter star's data is evident. 
The effect of averaging within the FGS on real data has been demonstrated. Successive tests indicate the data with binning at possible FGS averaging times. With good data (note these data were taken in FGS3 with 2/3 aperture-the best conditions we have at the moment) we should be able to detect and lock onto stars as faint as 15 th with the system as it presently exists.

iv. Clear vs 2/3 Aperture Comparison. Comparisons have been made between scans using a full aperture and a 2/3 aperture stop. A 2/3 aperture observation produces a reasonably "clean" S-Curve while the full aperture produces a degraded signal/noise ratio, or poor modulation in the S-Curve.

v. Change in tilt and decenter of the secondary mirror.

The initial transfer functions observed showed the following characteristic: The transfer function in FGS 3 yields best modulation in the signal. The signal peak is almost to the expected amplitude (about 7 on a normalized scale) for a fully functional FGS. The transfer functions in the other FGSs show greatly reduced modulation (around 0.2), FGS2 being far worse than FGS1 or 3. The poor performance was ascribed to off-axis coma and astigmatism. A mirror correction was made to correct for the inferred astigmatism and coma. The amplitudes are now more consistant with each other, ranging from 0.4 to 0.6 under different conditions for the different FGSs. The amplitude on FGS3 was in fact slightly reduced after the mirror moves.

\subsubsection{Summary of the Current Status of the FGSs. The HST is being shaken by solar} array thermal shock as it passes through the earth's terminator with sporadic thermal aftershock occurring during orbit day. The thermal gradients are causing "vehicle jitter" resulting in disturbances around the $0.1 \mathrm{~Hz}$ and $0.6 \mathrm{~Hz}$ modes, with amplitudes up to 0.2 arcseconds peak-to-peak initially through the terminator. The jitter is making the use of Fine Lock difficult-to-impossible on fainter stars, and making the interpretation of the transfer function scans in the FGSs difficult. However, engineering solutions to the jitter problem are being sought. The first implementation is due around the middle of October.

Compared with an ideal, stationary transfer function of a point source (single unresolved star), the transfer functions obtained vary from nearly ideal to very complex and convoluted. Comparison between transfer functions near day/night transitions and during quiescent periods demonstrate that the jitter is perturbing the signature of the transfer functions, but that in some cases (FGS2), non-ideal transfer functions with multiple values exist, which are not influenced by jitter. We do not have enough information yet to determine whether the problem is an optical alignment/mirror figure problem in the main telescope or possible internal FGS problems. The solution to determining the nature of the problems we have observed is to make carefully controlled observations which will isolate one or another of these possibilities.

\section{Recommendations}

The recommendation is to establish a controlled experiment by observing the same star at the same focal position "across FGS boundaries", i.e., to obtain transfer functions of the star at nearly the same position in the field of view for each FGS. The entire test will be performed twice for "repeatability" while providing some protection against failed guide star acquisitions. The transfer scans will be performed throughout each pickle to determine intra FGS changes in the characteristics of the transfer functions.

The result of these and other considerations is the "9 points of light" test, to be performed around the end of November. Observations of a star will be made at nine points within each FGS. See figure 4. The points have been chosen to be sensitive to radial and transverse variations within each FGS and to interFGS variations due to the primary and 
secondary mirror optical distortions. 5 scans with both the clear and $2 / 3$ pupil filters will be performed at each position through each aperture. Assuming that at least 50 percent of the data are good, we then may have sufficient information to determine the source of the corrupted transfer signature, and/or whether they have either internal FGS dependence or OTA field dependence.

The second test to be performed in the November timeframe, will demonstrate the capability of the FGSs to perform astrometric measurements. This test was originally designed as a series of tests to check the engineering capabilities of the instrument, such as fundamental capabilities of each FGS to lock onto stars in Fine Lock with full aperture, utilization of different filters, determine whether or not each FGS is capable of measuring the position of a 17th magnitude star, and whether or not the FGSs are capable of performing meaningful transfer scans.

Our current plan is to perform these tests, to take whatever corrective action is indicated, and to repeat the tests until they are successful.

\section{Conclusion}

If the jitter fix is successful, and we are able to characterize the FGS/OTA problems, we eventually should be able to reach our original goal of 17 th magnitude with a few milliarcseconds (rms) relative pointing accuracy.

We hope that the successful combination of these tests will indicate that the FGSs are capable of performing milliarcsecond astrometry and that we will know which FGS to choose as the Astrometer. The tests should indicate adjustments that must be made before we can reach astrometric level performance. Only after successful completion of these tests can we then proceed to the Science Verification portion of our program, where milliarcsecond calibrations are performed.

\section{Acknowledgements}

This work is based on observations with the NASA/ESA Hubble Space Telescope, obtained in part at the Space Telescope Science Institute, which is operated by the Association of Universities for Research in Astronomy, Inc., under NASA contract NAS526555.

We gratefully acknowledge support from the National Aeronautics and Space Administration under contract NAS8-32906.

\section{Bibliography}

1. M. Froeschle and J. Kovalevsky, Astron. and Astrophys., 116, 89, 1982.

2. W. van Altena, "Astronomy," Ann. Rev. Astron. and Astrophys., 21, 131, 1983.

3. P. D. Hemenway, R. L. Duncombe,W. H. Jefferys and P. J. Shelus, "Using Space Telescope to Tie the HIPPARCOS and Extragalactic Reference Frames Together," in HIPPARCOS: Scientific Aspects of the Input Catalogue Preparation, T. D. Guyenne and J. Hunt, eds., European Space Agency Special Publication ESA SP-234, p. 261, 1985. 
4. W. H. Jefferys, G. F. Benedict, P. D. Hemenway, P. J. Shelus and R. L. Duncombe, "Prospects for Astrometry with the Hubble Space Telescope," Celestial Mechanics, 37, 299, 1985.

5. W. H. Jefferys, M. J. Fitzpatrick and B. E. McArthur, "GaussFit: A system for Least Squares and Robust Estimation," Celestial Mechanics, 41, 39, 1988.

6. R. L. Duncombe, W. H. Jefferys, G. F. Benedict, P. D. Hemenway and P. J.Shelus, "Expectations for Astrometry with the Hubble Space Telescope," Proceedings of International Astronomical Union Symposium Number 141, Pulkovo Observatory, Leningrad, Kluwer Acad. Pubs. 1990.

7. L. Abramowicz-Reed, A. Bradley, D. Story, G. Benedict, W. Jefferys, "Status of the Flight Hardware and Ground System for Hubble Space Telescope Astrometry," Poster paper presented to the American Astronomical Society, Washington, DC, January 9-13, 1990.

8. R. L. Duncombe, W. H. Jefferys, P. J. Shelus, P. D. Hemenway and G. F. Benedict, "Astrometry Using the Hubble Space Telescope Fine Guidance Sensors," COSPAR Advances in Space Research 1990, Pergamon Press, Oxford, 1991. 


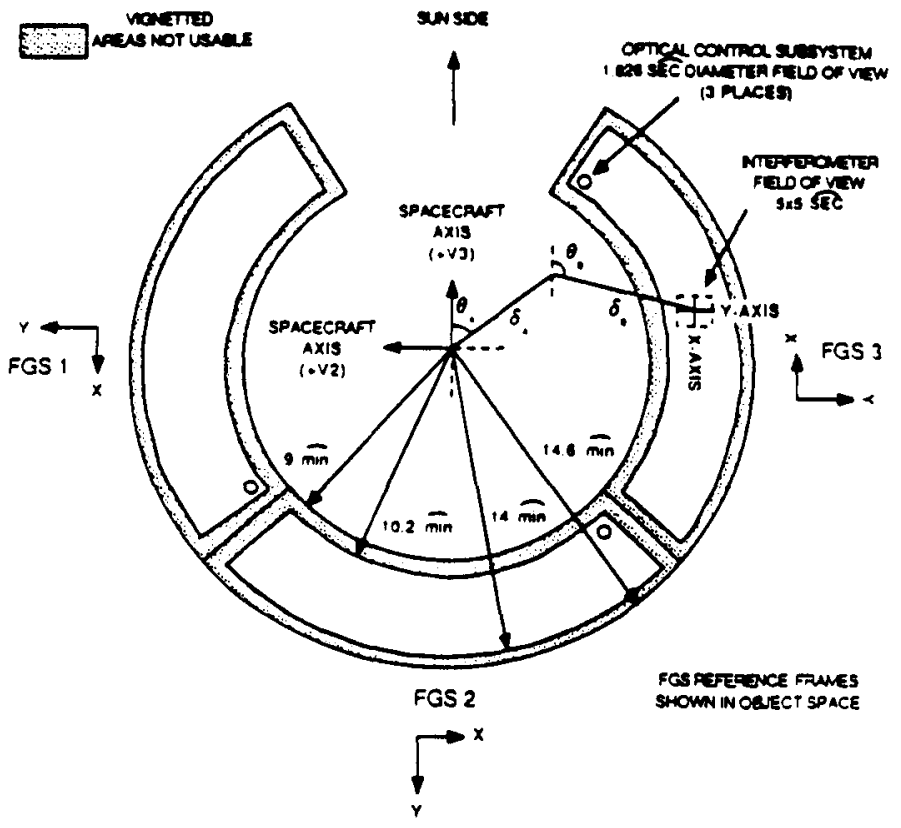

FGS FIELD OF VIEW PROJECTED

ONTO THE CELESTIAL SPHERE

FIGURE 1

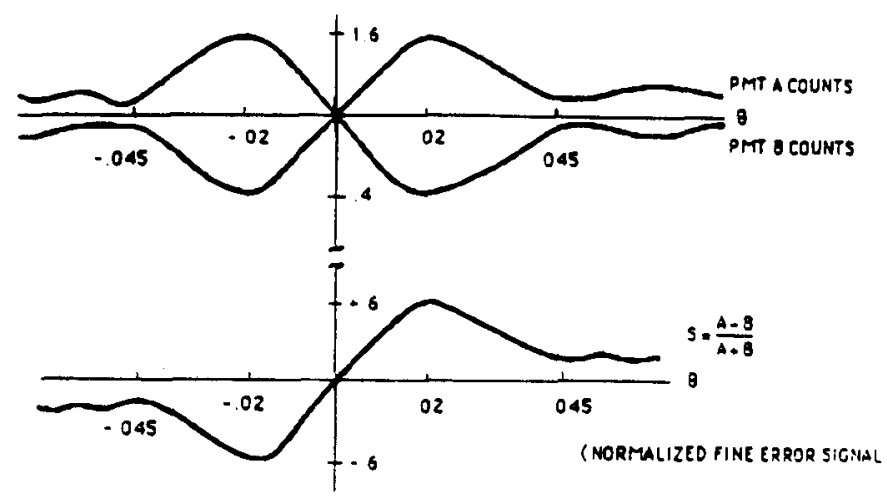

CONSTRUCTION OF A

HYPOTHETICAL S-CURVE

FIGURE 2 


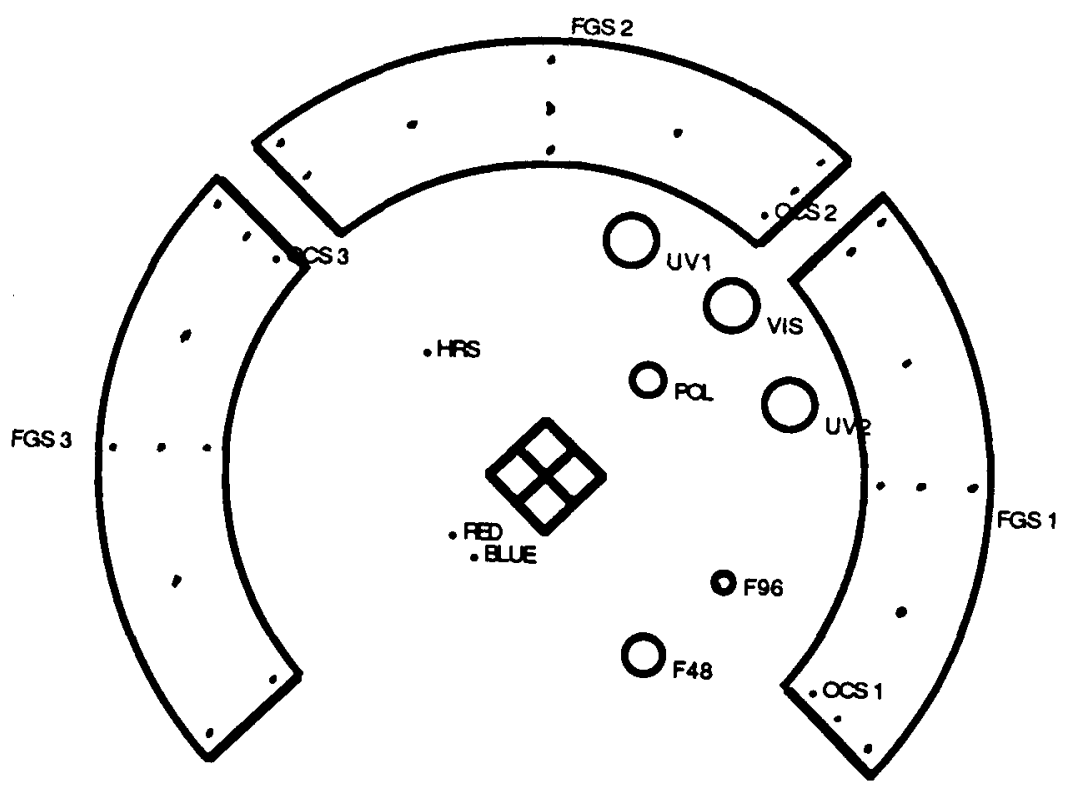

LOCATIONS OF THE

9 POIINTS OF LIGHT

OBSERVATIONS

FIGURE 4 
XXIS
AXIS
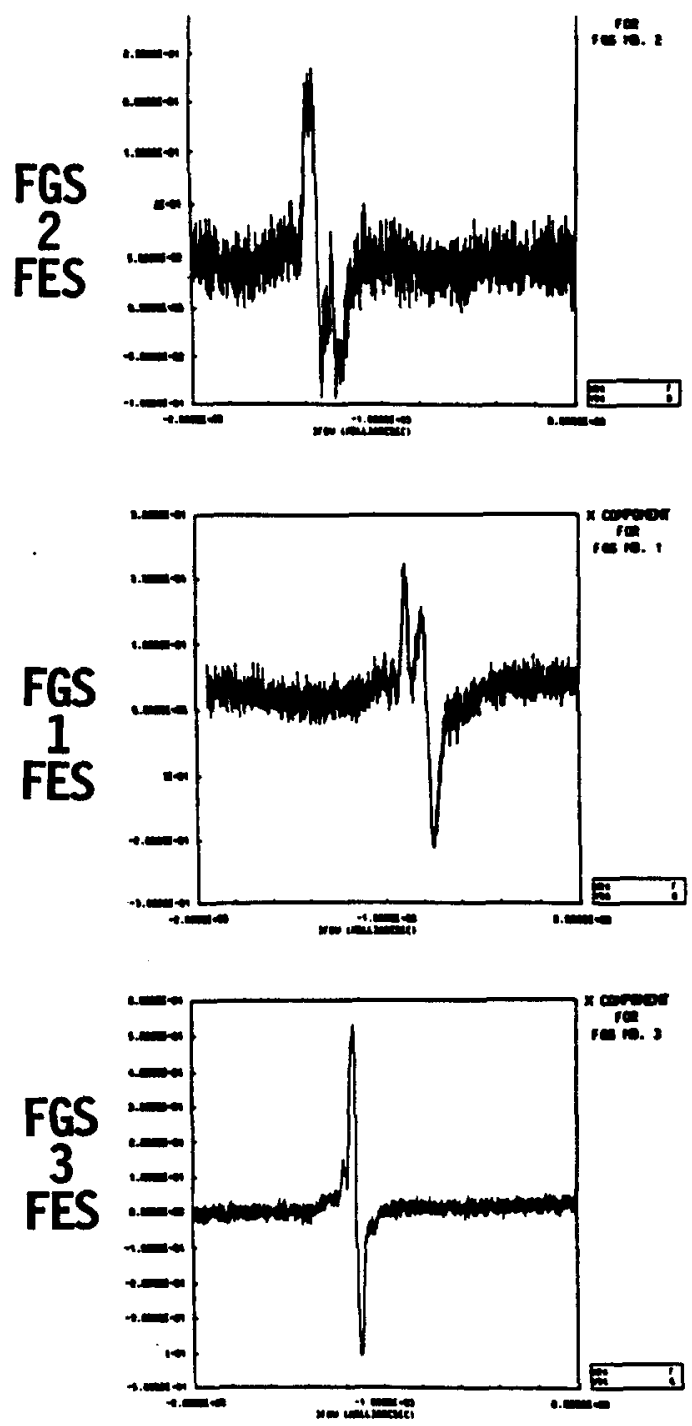

$\underset{\text { AXIS }}{Y}$
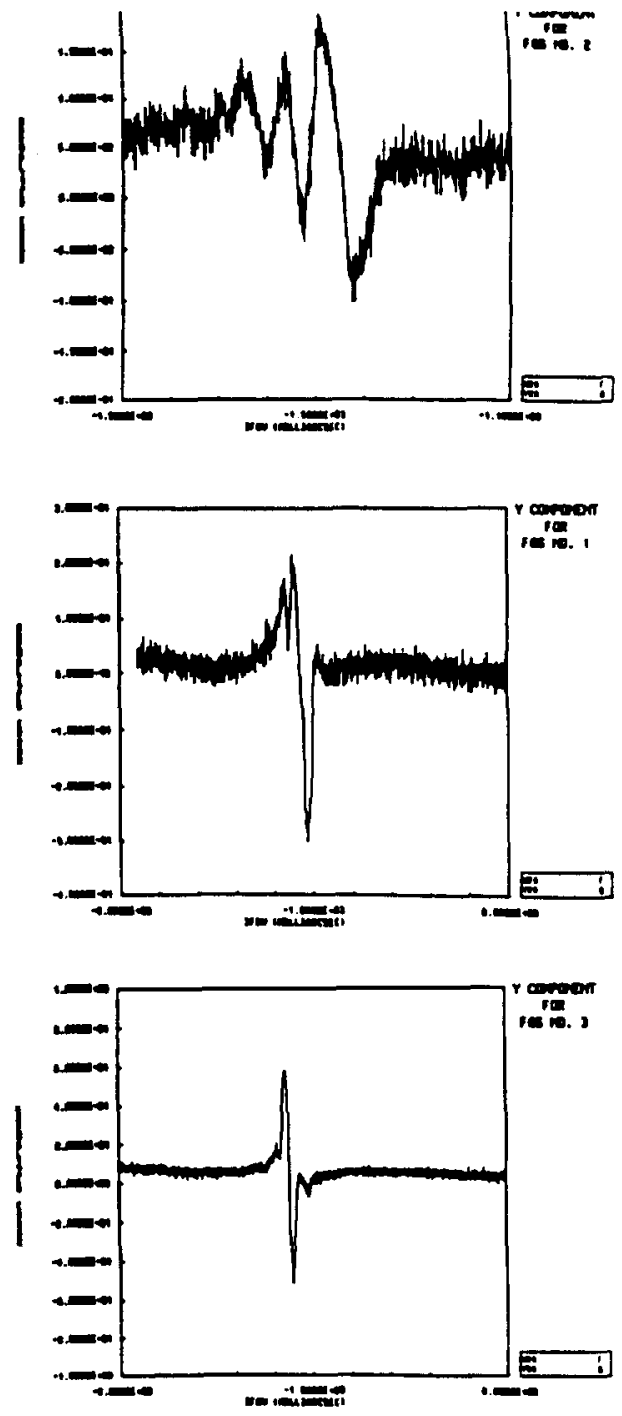

\section{$X$ AND $Y$ TRANSFER FUNCTIONS OBSERVED IN FGSs 2, 1, AND 3 (top to bottom)}

Normalized Fine Error Signal vs Positional Offset

FIGURE 3 